\title{
LETTERS
}

\section{Nurse anesthesiologists}

I read with interest the Analysis article on addressing the anesthesia needs of rural and remote communities in Canada. ${ }^{1}$

The US model of independent certified registered nurse anesthesiologists (CRNAs) provides the same level of perioperative care throughout the United States. CRNAs practise with the same anesthesia knowledge, same responsibilities and same outcomes as physicians. ${ }^{2-4}$ Southern Africa has nurse anesthesiology, and Denmark and France recognize that nurses can be valuable providers of perioperative care.

\section{Robert Bland MSN}

Certified registered nurse anesthesiologist, nonsurgical pain management-certified, Peace Harbor Medical Center, Florence, Ore.

- Cite as: CMAJ 2020 October 13;192:

E1214. doi: 10.1503/cmaj.76505

\section{References}

1. Orser BA, Wilson CR. Canada needs a national strategy for anesthesia services in rural and remote regions. CMAJ 2020;192:E861-3.

2. Bankert MA. Watchful care: a history of America's nurse anesthetists. Chicago: American Association of Nurse Anesthetists; 1989.

3. Certified registered nurse anesthetist fact sheet. Park Ridge (IL): American Association of Nurse Anesthetists; updated 2019 Aug. 8. Available: www.aana.com/patients/certified-registered-nurse -anesthetists-fact-sheet (accessed 2020 Sept. 16).

4. Dulisse B, Cromwell J. No harm found when nurse anesthetists work without supervision by physicians. Health Affairs 2010;29:1469-75.

Competing interests: None declared. 